\title{
Neural decoding of single vowels during covert articulation using electrocorticography
}

\section{Shigeyuki lkeda ${ }^{1 *}$, Tomohiro Shibata ${ }^{1,2}$, Naoki Nakano ${ }^{3}$, Rieko Okada $^{3}$, Naohiro Tsuyuguchi $^{4}$, Kazushi Ikeda ${ }^{1}$ and Amami Kato ${ }^{3,5}$}

${ }^{1}$ Graduate School of Information Science, Nara Institute of Science and Technology, Ikoma, Japan

${ }^{2}$ Graduate School of Life Science and Systems Engineering, Kyushu Institute of Technology, Kitakyushu, Japan

${ }^{3}$ Department of Neurosurgery, Kinki University Faculty of Medicine, Sayama, Japan

${ }^{4}$ Department of Neurosurgery, Graduate School of Medicine, Osaka City University, Osaka, Japan

${ }^{5}$ Core Research for Evolutionary Science and Technology, Japan Science and Technology Agency, Kawaguchi, Japan

\section{Edited by:}

John J. Foxe, Albert Einstein College of Medicine, USA

Reviewed by:

Antoine Shahin, The Ohio State

University, USA

Sundeep Teki, University College

London, UK

Travis White-Schwoch,

Northwestern University, USA

\section{*Correspondence:}

Shigeyuki Ikeda, Mathematical Informatics Laboratory, Graduate

School of Information Science, Nara Institute of Science and Technology, 8916-5 Takayama, Ikoma 630-0192, Japan

e-mail: shigeyuki-i@is.naist.jp
The human brain has important abilities for manipulating phonemes, the basic building blocks of speech; these abilities represent phonological processing. Previous studies have shown change in the activation levels of broad cortical areas such as the premotor cortex, the inferior frontal gyrus, and the superior temporal gyrus during phonological processing. However, whether these areas actually convey signals to representations related to individual phonemes remains unclear. This study focused on single vowels and investigated cortical areas important for representing single vowels using electrocorticography (ECoG) during covert articulation. To identify such cortical areas, we used a neural decoding approach in which machine learning models identify vowels. A decoding model was trained on the ECoG signals from individual electrodes placed on the subjects' cortices. We then statistically evaluated whether each decoding model showed accurate identification of vowels, and we found cortical areas such as the premotor cortex and the superior temporal gyrus. These cortical areas were consistent with previous findings. On the other hand, no electrodes over Broca's area showed significant decoding accuracies. This was inconsistent with findings from a previous study showing that vowels within the phonemic sequence of words can be decoded using ECoG signals from Broca's area. Our results therefore suggest that Broca's area is involved in the processing of vowels within phonemic sequences, but not in the processing of single vowels.

Keywords: covert articulation, single vowel, neural decoding, electrocorticography (ECoG), functional mapping

\section{INTRODUCTION}

Language appeared during the course of human evolution, enabling us to communicate with others. In oral communication, speech consists of complicated sounds which are rarely found in other animals. Phonemes are the basic building blocks that make up speech. The human brain can flexibly manipulate phonemes to compose and decompose syllables or words, in what is known as phonological processing (McBride-Chang, 1996). Previous studies have reported that broad cortical areas such as the premotor cortex, the inferior frontal gyrus (IFG), and the superior temporal gyrus (STG) show changes in activation levels during phonological processing (Vigneau et al., 2006). However, the question remains as to whether these areas actually convey signals on the phoneme-related representations needed for manipulating phonemes during phonological processing.

Decoding-based approaches provide sophisticated methods for identifying cognitive or perceptual states from brain activity (Haynes and Rees, 2006). Recent studies have demonstrated that phoneme-related representations can be extracted from brain activity during covert speech, which is assumed to include all language processes other than the motor execution stage. Some studies have reported successful identification of vowels using electroencephalography (EEG) (DaSalla et al., 2009) or electrocorticography (ECoG) (Leuthardt et al., 2011). However, those studies did not localize cortical areas for the identification of vowels. Pei et al. (2011) reported that phonemes within words could be identified using ECoG signals measured during covert speech of those words, and they localized cortical areas carrying information for the identification of phonemes. They decoded vowels within the phonemic sequence of words using ECoG signals. However, neural substrates for the representation of phonemes may differ when a vowel is processed within a phonemic sequence and when it is processed alone. Addressing this issue contributes to the understanding of brain function which manipulates basic speech sounds.

To this end, we measured ECoG signals while subjects covertly articulated single vowels. Based on decoding accuracies using these ECoG signals, cortical areas in which brain activity discriminated single vowels were identified. Cortical areas such as the premotor cortex and STG were identified; these cortical areas matched previous findings (Pei et al., 2011). STG is involved in covert speech production, which is evident in that cortical activations increased over STG during covert word production (Pei et al., 2010). The premotor cortex is assumed to be important for articulatory planning (Duffau et al., 2003), and pronounced activations over the premotor cortex were found during covert 


\section{Subject 1}

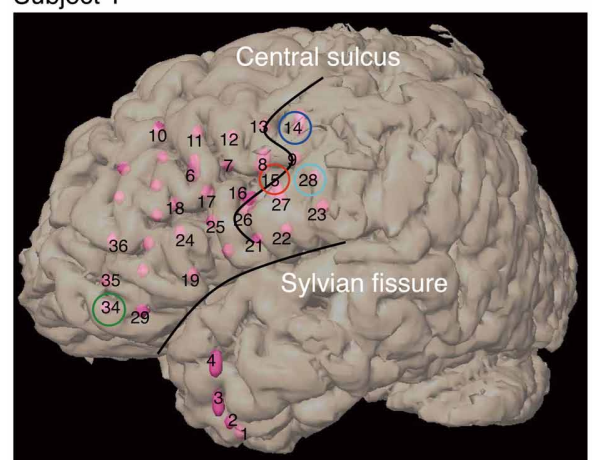

Dysesthesia around mouth

Speech arrest

Right facial spasm (around mouth) $\bigcirc$ Epileptic seizure

\section{Subject 2}

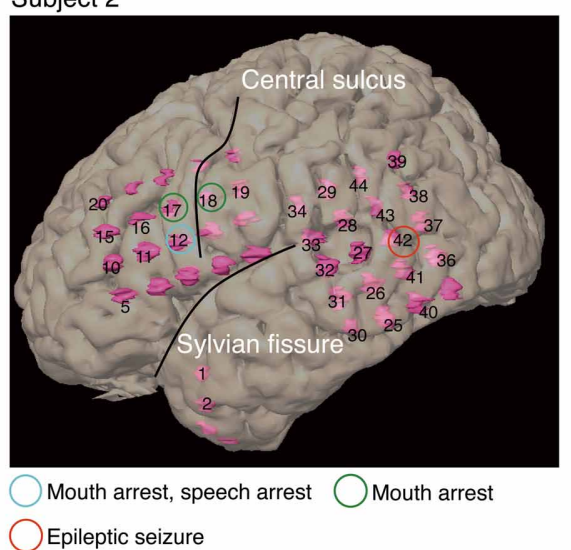

Epileptic seizure
Subject 3

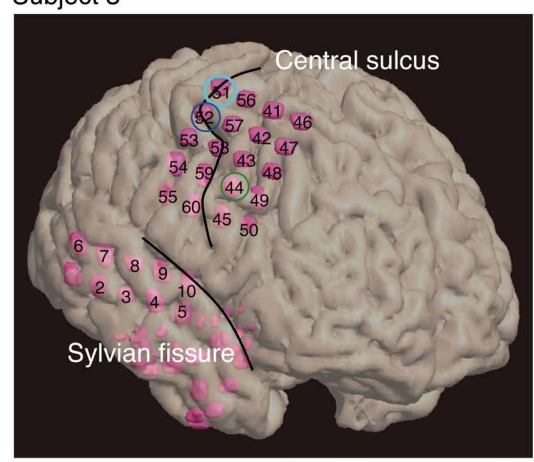

Left hand response Mouth response

Left hand spasm Epileptic seizure

Subject 4

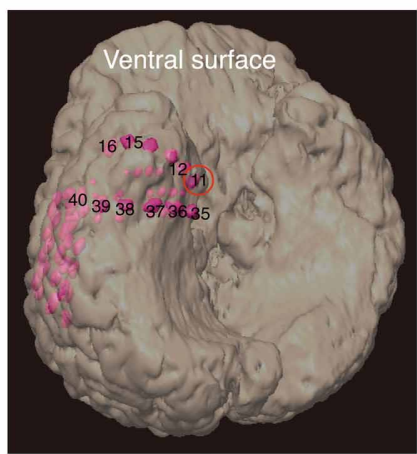

Amygdala

$\begin{array}{llllll}22 & 21 & 20 & 19 & 18 & 17\end{array}$

Anterior hippocampus

$\begin{array}{llllll}28 & 27 & 26 & 25 & 24 & 23\end{array}$

Posterior hippocampus

$\begin{array}{llll}34 & 33 & 32(31)(30) & 29\end{array}$

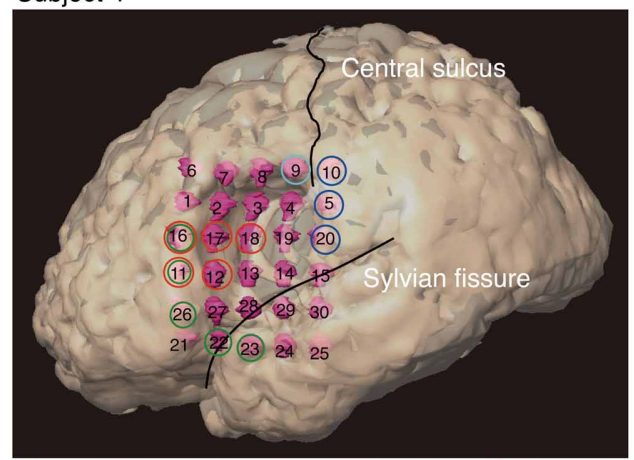

Right facial motor arrest

Speech arrest, paralexia, naming deficit

spasm (right side of tongue, around mouth) $\bigcirc$ Epileptic seizure

FIGURE 1 | Electrode locations and responses to electrical stimulation for each subject, superimposed onto positions of the implanted subdural electrodes. The two black lines delineate the locations of the central sulcus and Sylvian fissure. Pink objects represent the electrodes. Unnumbered electrodes are those that were not used in this analysis due to severe measurement noise. Each colored circle indicates a response to electrical stimulation at the corresponding electrode location. Subject 3 had electrodes (11-40) over the right ventral temporal lobe. To facilitate visualization, electrodes 17-34 are not superimposed on the ventral surface shown in the figure. Electrodes 17-22 are shown over the amygdala, electrodes 23-28 over the anterior hippocampus, and electrodes 29-34 over the posterior hippocampus.

over Broca's area showed significant decoding accuracies. This was considered to be because Broca's area is assumed to be important for the segmentation of words into individual phonemes as a part of phonological processing (Zatorre et al., 1992, 1996; Burton et al., 2000). Our results suggest that Broca's area is involved in the 
processing of vowels within phonemic sequences, but not in the processing of single vowels.

\section{MATERIALS AND METHODS}

\subsection{SUBJECTS}

Four subjects with intractable epilepsy (one male, three females) participated in our investigation. The subjects underwent temporary placement of subdural electrodes to localize seizure foci before surgical treatment of their epilepsy. All subjects provided written informed consent to participate in this study. All study protocols were approved by the ethics committees of both Kinki University Faculty of Medicine (21-135) and Nara Institute of Science and Technology (2203).

Individual subjects had subdural electrodes placed over the frontal, parietal, and temporal regions (Figure 1). Only Subject

\begin{tabular}{|c|c|c|c|c|}
\hline Subject & Age & Sex & $\begin{array}{l}\text { Language } \\
\text { dominance }\end{array}$ & $\begin{array}{l}\text { Electrode } \\
\text { location }\end{array}$ \\
\hline 1 & 17 & M & Left & $\begin{array}{l}\text { Left } \\
\text { frontal-parietal-temporal }\end{array}$ \\
\hline 2 & 38 & $\mathrm{~F}$ & Right and left & $\begin{array}{l}\text { Left } \\
\text { frontal-parietal-temporal }\end{array}$ \\
\hline 3 & 34 & $\mathrm{~F}$ & Left & $\begin{array}{l}\text { Right } \\
\text { frontal-parietal-temporal- } \\
\text { amygdala-hippocampus }\end{array}$ \\
\hline 4 & 22 & $\mathrm{~F}$ & Right and left & $\begin{array}{l}\text { Left } \\
\text { frontal-parietal-temporal }\end{array}$ \\
\hline
\end{tabular}

Table 2 | Sites with positive findings identified via electrical stimulation.

\begin{tabular}{lcl}
\hline & $\begin{array}{c}\text { Electrode } \\
\text { number }\end{array}$ & Responses \\
\hline Subject 1 & 14 & Right facial spasm (around mouth) \\
& 28 & Epileptic seizure \\
& 34 & Dysesthesia around mouth \\
Speech arrest
\end{tabular}

3 had electrodes placed on the amygdala and anterior and posterior hippocampus. Electrodes on all subjects except Subject 3 were localized over the left hemisphere, and electrodes on all subjects were based solely on the requirements of clinical evaluations, without any alterations made for endpoints of this study.

We conducted a Wada test for subjects to specify the hemispheric dominance for language (Wada and Rasmussen, 1960). As shown in Table 1, the left hemisphere was dominant in Subjects 1 and 3, and both hemispheres were dominant in Subjects 2 and 4 .

Because we postoperatively preserved function in cortical areas for each subject, we performed electrical stimulation mapping (ESM), which enabled us to localize epileptic foci for each subject and to identify critical sensory, motor or language areas. Sites with positive findings by electrical stimulation are shown in Table 2.

In three of the four subjects, we found electrodes in which electrical stimulation caused a disruption of speech (Speech arrest) (Table 2). We defined the cortical areas showing "Speech arrest" as Broca's area. Note that the cortical area on which electrode 12 in Subject 2 was placed was assumed not to be Broca's area because the cortical area was close to the central sulcus. In addition, because electrode 23 in Subject 4 was placed on the temporal part, this area was not Broca's area.

\subsection{EXPERIMENTAL SETUP}

Each subject performed a task in this study while sitting upright in a hospital bed. Figure 2 illustrates the task procedure. Each trial consisted of a presentation and a blank period. The presentation period lasted $200 \mathrm{~ms}$, and the blank period lasted $1100 \mathrm{~ms}$, so each trial lasted $1300 \mathrm{~ms}$ overall. We selected three of the five Japanese vowels because the task was designed to be as brief as possible, in accordance with the medical judgment of the doctors collaborating in this study. A task consisted of 90 trials, with 30 for each vowel. Each task thus lasted about 120 s. Each vowel was shown on a liquid crystal display monitor during the presentation period, and the subjects were asked to covertly articulate the vowel one single time during the blank period without intentional movements such as of the lips or tongue. The three vowels selected were /a/, /i/, and/u/ (/a/:/あ/, /i/:/ /, and /u/:/う/) Each was presented as a white hiragana letter against a black background. The order

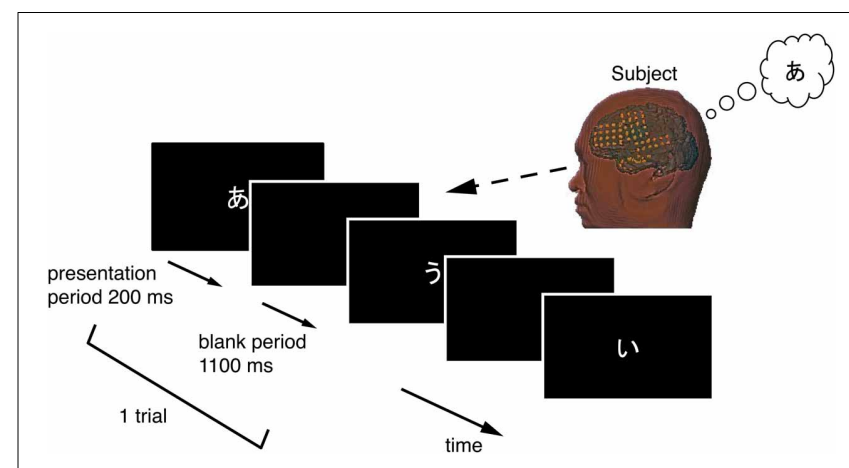

FIGURE 2 | Temporal sequence in the vowel-articulating task. The solid arrow indicates the time-line. Each trial consisted of a presentation period followed by a blank period. The subject covertly articulated the vowel one single time during the blank period. 


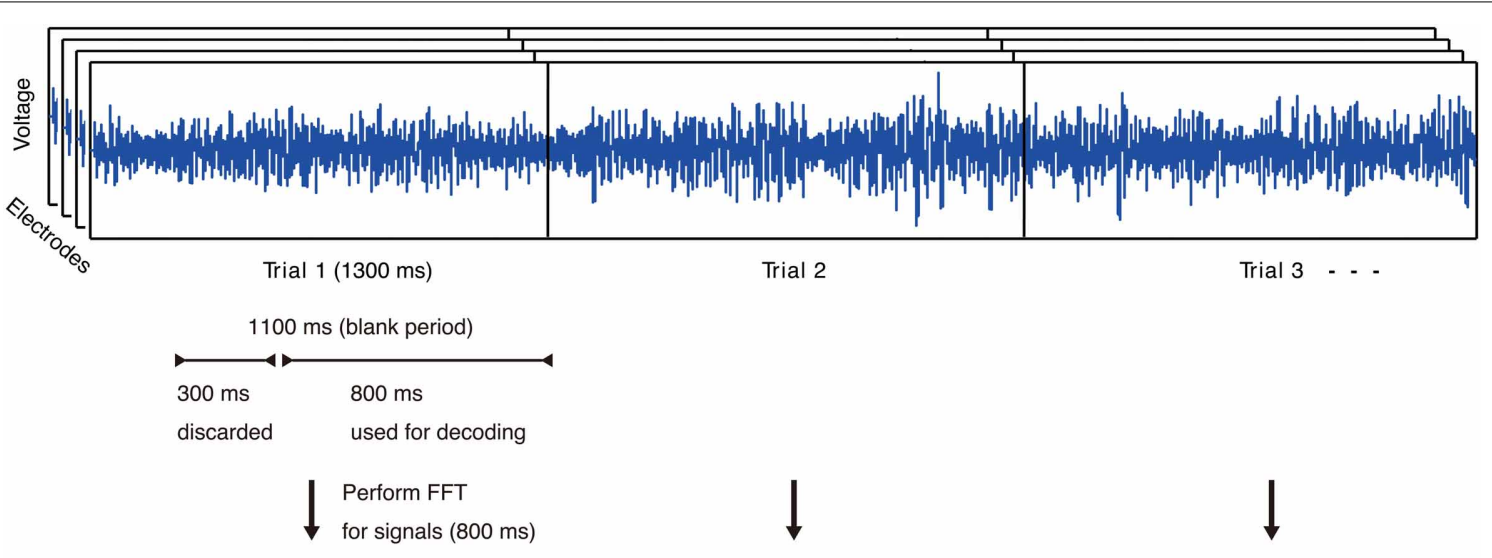

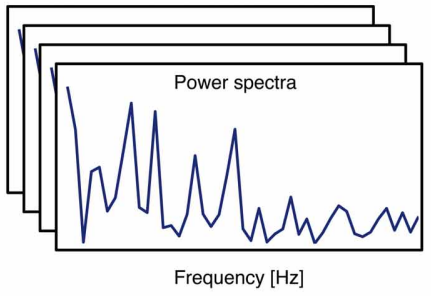

Frequency $[\mathrm{Hz}]$

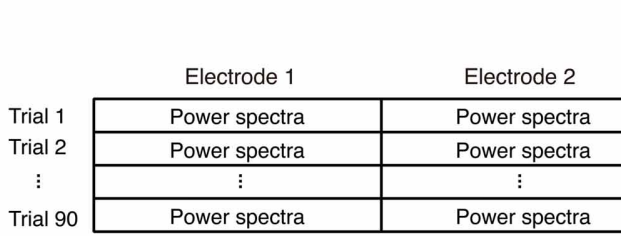

FIGURE 3 | Feature extraction process using ECoG signals. Top: Representative ECOG signals recorded in individual trials. The ECoG signals recorded in an early stage of individual trials could reflect immediate responses to visual stimuli. In order to decode single vowels based on information from covert articulation, and not
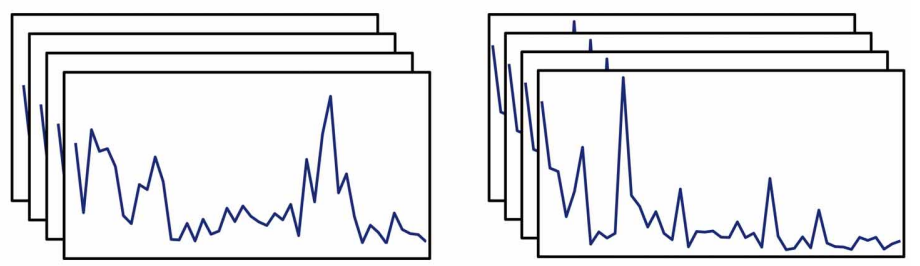

Dataset for decoding

\begin{tabular}{|c|c|c|}
\hline \multicolumn{2}{c}{} & Electrode N \\
\hline & $\ldots$ & Power spectra \\
\hline & $\ldots$ & Power spectra \\
\hline & $\ldots$ & Power spectra \\
\hline
\end{tabular}

information from the visual stimuli, we used ECoG signals arriving only after $300 \mathrm{~ms}$ into the blank period. Middle: Representative power spectra extracted from each trial and each electrode. Bottom: For each trial, power spectra from all electrodes. $\mathrm{N}$ is the number of electrodes on an individual subject. of presentation was randomized so that subjects were unable to predict which vowel would be presented in a given trial.

\subsection{DATA COLLECTION}

UZN-series electrodes (Unique Medical Corporation, Tokyo, Japan) were used, and signals from the electrodes were recorded using an EEG1000-series measurement system (Nihon Kohden Corporation, Tokyo, Japan). Intra-electrode distance was $10 \mathrm{~mm}$, and the sampling frequency for analog-to-digital conversion was $1000 \mathrm{~Hz}$. All electrodes were referenced to a scalp electrode placed on the nasion. In all subjects, electrodes containing severe measurement noise were removed from the analysis. These electrodes are shown as unnumbered electrodes (Figure 1). All data were analyzed using Matlab 2011a software (The MathWorks, Natick, MA, USA).

\subsection{FEATURE EXTRACTION}

The ECoG signals recorded in an early stage of individual trials could reflect immediate responses to visual stimuli. In order to decode single vowels based on information from covert articulation, and not information from the visual stimuli, we used
ECoG signals arriving only after $300 \mathrm{~ms}$ into the blank period. (Figure 3).

For ECoG features as input to decoding analyses, we focused on the high-gamma frequency band of ECoG signals (70$110 \mathrm{~Hz}$ ). The high-gamma band is assumed to be associated with auditory perception of phonemes or word production (Crone et al., 2001a,b; Canolty et al., 2007; Pei et al., 2010). For each electrode, the power spectra in the highgamma bands were extracted from ECoG signals in the blank period of individual trials using fast Fourier transform (FFT) (Figure 3). With selection of the high-gamma band, we avoided the $60-\mathrm{Hz}$ power noise and associated harmonics (e.g. $120 \mathrm{~Hz}$ ). The power spectra were used as feature vectors for decoding analyses.

For normalization of power spectra, we divided all trials into testing trials and training trials, and then calculated $\mathrm{z}$-scores from each power spectrum across the training trials for individual electrodes. To normalize testing trials without using those trials, we calculated $\mathrm{z}$-scores from each power spectrum across the testing trials using the mean and standard deviation calculated from each power spectrum across the training trials. 


\section{High-gamma band}

Subject 1

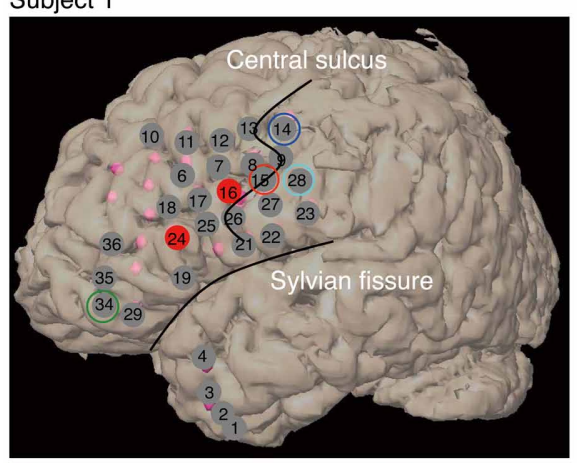

Dysesthesia around mouth

Speech arrest

$\bigcirc$ Right facial spasm (around mouth) $\bigcirc$ Epileptic seizure

\section{Subject 2}

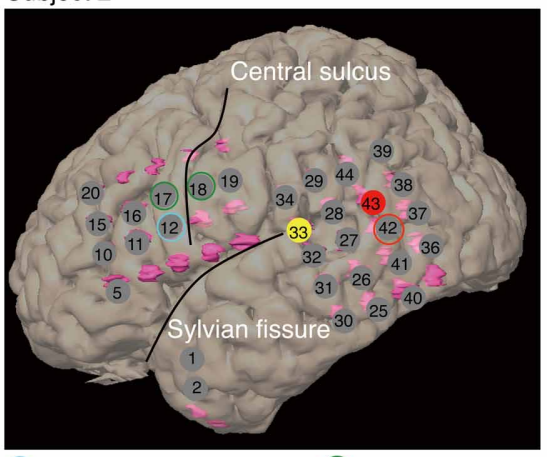

Mouth arrest, speech arrest $\bigcirc$ Mouth arrest

Epileptic seizure

\section{Subject 3}

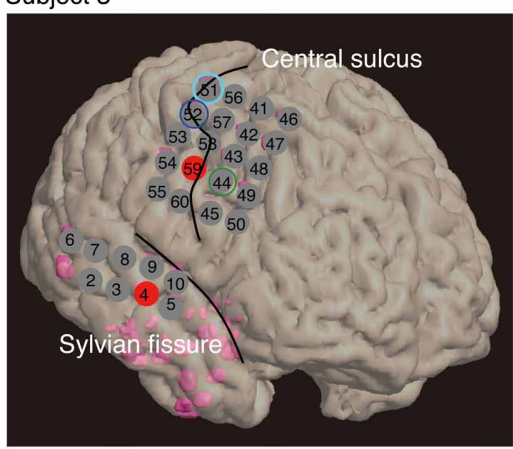

Left hand response $\bigcirc$ Mouth response

Left hand spasm Epileptic seizure

\section{Subject 4}

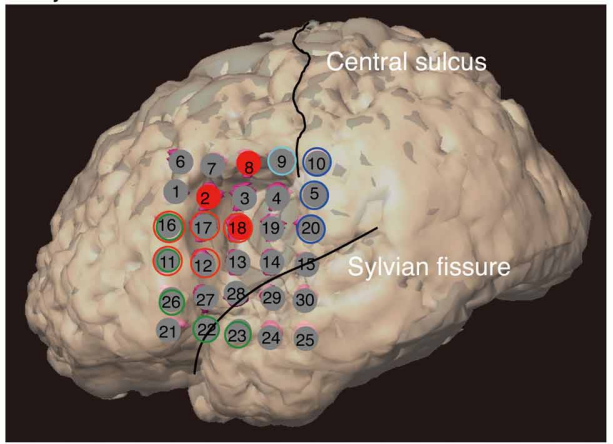

Right facial motor arrest

Speech arrest, paralexia, naming deficit

spasm (right side of tongue, around mouth) $\bigcirc$ Epileptic seizure

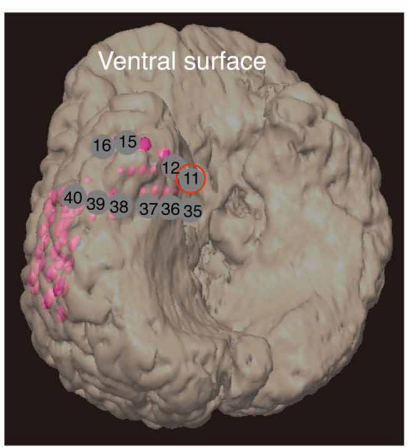

Amygdala 22-21-20-19-18-17

Anterior hippocampus 28-27.26-25-24-23

Posterior hippocampus 34- 33- 32-31- $30 \cdot-29$
FIGURE 4 | Decoding accuracies acquired from the high-gamma power spectra for each electrode in individual subjects. We estimated the decoding accuracy of each electrode by 15 -fold cross-validation. Decoding accuracies were superimposed onto the electrode map of each subject. To facilitate visualization, for Subject 3, electrodes 17-34 are not superimposed onto the ventral surface shown in the figure. The color filling each circle represents the level of significance of the decoding accuracy; $p$-values corresponding to decoding accuracy were computed from a distribution given by the normal approximation to the binomial distribution (mean: nc, standard deviation: $\sqrt{n c(1-c)}, n$ : total number of trials, $c$ : chance level 0.33 ).

\subsection{EVALUATION}

We constructed a linear classifier (decoder) to classify vowels from feature vectors on a trial-by-trial basis. The decoder calculated the linearly weighted sum of the features plus a bias for each class (/a/, /i/, and $/ \mathrm{u} /$ ), and the class with the maximum value was chosen as the classified class. Individual weights and biases were determined using a support vector machine (SVM) with the linear kernel applied to the training trials (Vapnik, 1998). SVM is a commonly 
used algorithm in the field of brain reading (Haynes and Rees, 2006). We used LIBSVM (Chang and Lin, 2011) in Matlab to implement the SVM.

To evaluate decoding accuracy, we performed a crossvalidation approach using all of the trials. In this approach, all of the trials were divided into 15 subgroups; 14 of the subgroups were used for training a classifier, and the remaining subgroup was used for testing the classifier. This procedure was repeated 15 times, using all of the trials of all of the subgroups as testing trials once (15-fold cross-validation). Decoding accuracy was calculated as a percentage of the correct classifications.

For each subject, we computed the decoding accuracy from each electrode, and derived a $p$-value corresponding to each decoding accuracy from a distribution given by the normal approximation to the binomial distribution. The mean of the distribution is $n c$ ( $n$ : total number of trials, $c$ : chance level 0.33 ); standard deviation is calculated according to $\sqrt{n c(1-c)}$. We then determined cortical representation areas of vowels in covert articulation based on decoding accuracies significantly greater $(p<0.05)$ than the level of chance.

\section{RESULTS}

To identify cortical areas in which brain activity discriminates single vowels of covert articulation, we statistically evaluated decoding accuracies from individual electrodes in each subject. Decoding accuracies corresponding to the electrodes were superimposed onto the electrode map of each subject (Figure 4). In addition, Table 3 showed over which cortical areas statistically significant electrodes were placed. The results shown in Table 3 demonstrated that the cortical areas able to discriminate single vowels were the primary motor area, the premotor cortex, STG, and so on. Decoding accuracies from these areas ranged from 42.2 to $46.7 \%$ (chance level 33.3\%). Furthermore, we found that no electrodes over Broca's area showed significant decoding accuracies (Table 4).

Table 3 | Cortical areas showing significant decoding accuracies in use of the high-gamma power spectra.

\begin{tabular}{|c|c|c|c|}
\hline & $\begin{array}{l}\text { Electrode } \\
\text { number }\end{array}$ & Cortical area & $\begin{array}{l}\text { Accuracy } \\
(\%)(p)\end{array}$ \\
\hline \multirow[t]{2}{*}{ Subject 1} & 16 & Left primary motor area & $42.2(p<0.05)$ \\
\hline & 24 & Left premotor cortex & $42.2(p<0.05)$ \\
\hline \multirow[t]{2}{*}{ Subject 2} & 33 & Left superior temporal gyrus & $46.7(p<0.005)$ \\
\hline & 43 & Left angular gyrus & $42.2(p<0.05)$ \\
\hline \multirow[t]{2}{*}{ Subject 3} & 4 & Right superior temporal gyrus & $44.4(p<0.05)$ \\
\hline & 59 & $\begin{array}{l}\text { Right primary somatosensory } \\
\text { area }\end{array}$ & $42.2(p<0.05)$ \\
\hline \multirow[t]{3}{*}{ Subject 4} & 2 & $\begin{array}{l}\text { Left dorsolateral prefrontal } \\
\text { cortex }\end{array}$ & $42.2(p<0.05)$ \\
\hline & 8 & Left premotor cortex & $42.2(p<0.05)$ \\
\hline & 18 & Left premotor cortex & $42.2(p<0.05)$ \\
\hline
\end{tabular}

\section{DISCUSSION}

Our purpose was to identify cortical areas in which brain activity can discriminate single vowels of covert articulation. We identified cortical areas such as the premotor cortex and STG (Figure 4); these cortical areas were consistent with the previous findings (Pei et al., 2011). We also found that no electrodes over Broca's area showed significant decoding accuracies (Table 4), whereas Pei et al. (2011) reported that ECoG signals from the area carry information about the discrimination of vowels within phonemic sequences. This difference could be attributed to the function of Broca's area. When a subject covertly articulates a word, the word is segmented in this area into individual phonemes as a part of phonological processing (Zatorre et al., 1992, 1996; Burton et al., 2000). In addition, the high-gamma power increased over Broca's area during segmentation of words (Herman et al., 2013). Therefore, in the previous study (Pei et al., 2011), individual vowels within phonemic sequences may have been decoded using the high-gamma band from Broca's area, which contains information about individual vowels segmented from words. Subjects in our study covertly articulated only single vowels, which thus did not require segmentation by Broca's area. This is why single vowels could not be decoded using the high-gamma band from Broca's area.

Since previous findings reported that cortical activations in the theta $(4-7 \mathrm{~Hz})$, alpha $(8-13 \mathrm{~Hz})$, beta $(14-30 \mathrm{~Hz})$ bands are associated with language processing (Bastiaansen and Hagoort, 2006; Giraud and Poeppel, 2012), we investigated cortical areas important for representation of single vowels when using these bands for decoding. Table 5 showed over which cortical areas statistically significant electrodes were placed. We found significant decoding accuracies in Broca's area when using the alpha or beta power spectra. This was expected because a previous study suggested that Broca's area has a motoric function which translates speech into articulatory code (Hickok and Poeppel, 2004). Furthermore, event-related desynchronization (ERD) occurred in alpha and beta bands over Broca's area during silent reading of words (Goto et al., 2011). Because ERD is assumed to include motor-related information (Crone et al., 1998), ECoG signals in the alpha and beta bands over Broca's area may contain information about the articulatory code of single vowels. Therefore, our results suggest that when subjects covertly articulate a single vowel, ECoG signals over Broca's area contain information about covert articulation of

Table 4 | Decoding accuracies in Broca's area using the high-gamma power spectra.

\begin{tabular}{lccc}
\hline & $\begin{array}{c}\text { Electrode } \\
\text { number }\end{array}$ & Accuracy (\%) & $\boldsymbol{p}$-value \\
\hline Subject 1 & 34 & 33.3 & 0.5 \\
Subject 2 & NA & & \\
Subject 3 & NA & 32.2 & 0.59 \\
Subject 4 & 11 & 40.0 & 0.09 \\
& 16 & 25.6 & 0.94 \\
& 22 & 30.0 & 0.75
\end{tabular}


Table 5 | Cortical areas showing significant decoding accuracies in use of the theta, alpha, and beta power spectra.

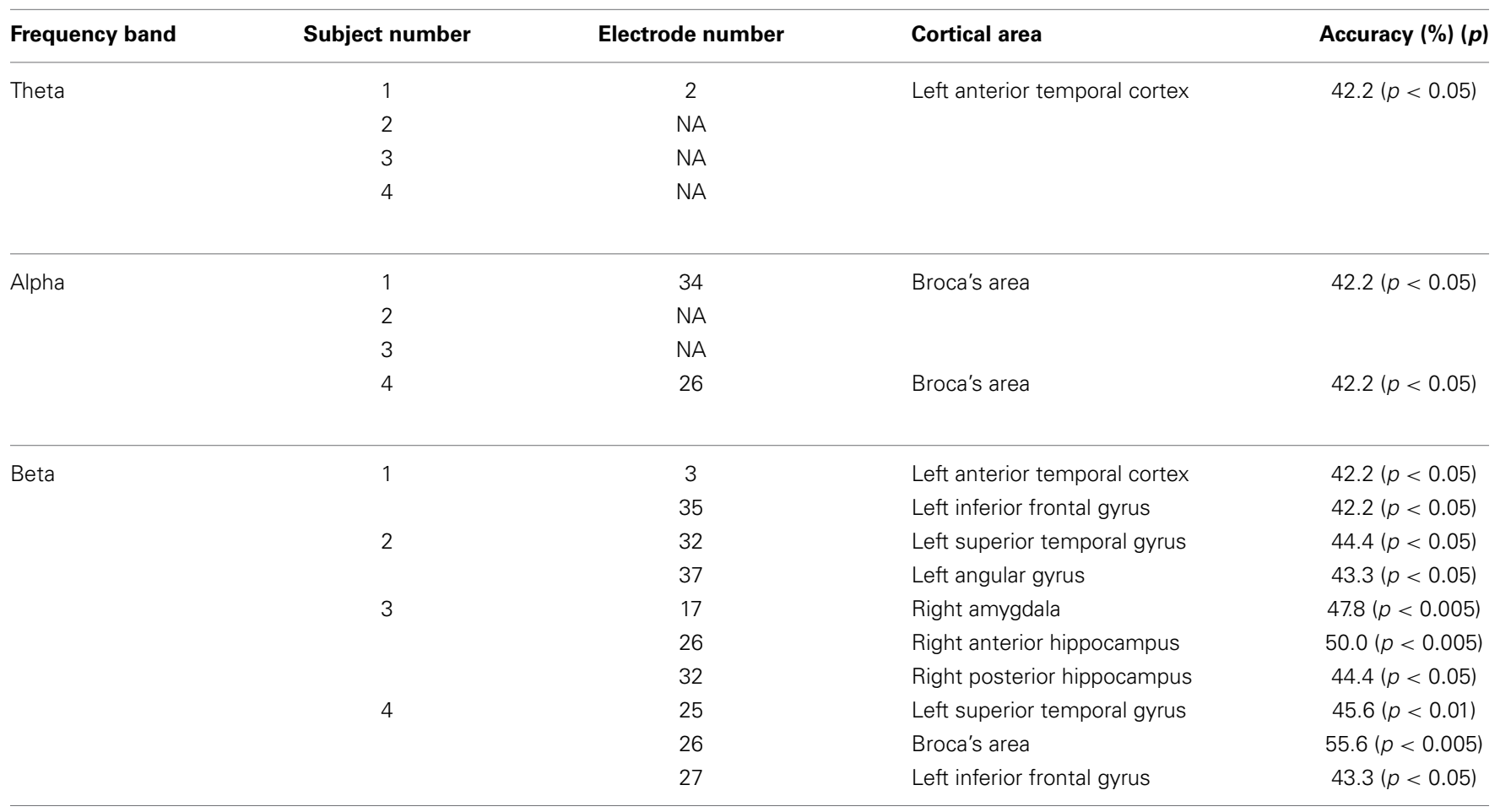

a single vowel, but not about segmentation of a phoneme sequence.

For the high-gamma band (Figure 4), significant decoding accuracies were seen in the primary motor area. We speculated that these results are associated with motor imagery. Motor imagery is usually performed unconsciously during movement preparation (Lotze and Halsband, 2006). Various studies, such as (Sharma et al., 2008; Miller et al., 2010), have reported that the primary motor area is activated during motor imagery tasks. In addition, (Wildgruber et al., 1996) suggested that covert speech is associated with motor imagery. In contrast, other studies have found that the primary motor cortex is barely activated during covert speech (Palmer et al., 2001; Huang et al., 2002), and also that individual vowels within words cannot be decoded using ECoG signals from the area (Pei et al., 2011). Given that words used in the previous study consisted of more than three phonemes, movement preparation for covert articulation of a word is assumed to be more complicated than for that of a single vowel. ECoG signals during covert articulation of a word thus carry information about complicated movement preparation. The present results suggest that decoding individual vowels within words using ECoG signals may be more difficult than decoding single vowels using ECoG signals during covert articulation of single vowels.

Some of the other areas important for the representation of single vowels were consistent with previous findings (Pei et al., 2011); these areas were the premotor cortex and STG (Table 3 ). The premotor cortex is assumed to be associated with motor planning (Duffau et al., 2003) and is activated not only during overt speech, but also during covert speech (Price, 2012). Single vowels may plausibly be decodable using ECoG signals from the premotor cortex. Regarding STG, some studies have provided evidence that this area is important for representing phonetic contents (Obleser et al., 2006; Chang et al., 2010; Leuthardt et al., 2011). This area might therefore represent auditory images of individual vowels while subjects are covertly articulating single vowels. In addition, previous findings revealed anatomical connectivity between the premotor cortex and STG (Saur et al., 2008); the connections are involved in a dorsal stream which is important for mapping sound to articulation.

\section{LIMITATIONS}

The subjects in this study were intractable epilepsy patients with a limited amount of time to participate in the experiment. Therefore, our experimental design did not include control conditions (i.e. using the same vowels but without covert speech and using non-language stimuli) to extract ECoG signals about covert articulation and vowel perception. Note that since we used ECoG signals arriving only after $300 \mathrm{~ms}$ into the blank period as input for decoding analyses, input used for decoding is assumed not to contain information from visual stimuli. This suggests that our ability to decode single vowels was based on information of neural processes involved in covert articulation, rather than information of processes involved in visual stimuli.

In this study, we concluded that Broca's area is involved in the processing of vowels within phonemic sequences, but not in processing single vowels. To confirm our conclusion, we should investigate whether vowels within the phonemic sequence of words can be decoded using ECoG signals from Broca's area. Due to their time constraints, subjects in this study were unable to perform the task of covert articulation of vowels within phonemic sequences. 


\section{ACKNOWLEDGMENTS}

We wish to thank Dr. T. Kubo and Dr. S. Nishida for valuable comments on the manuscript. This work was supported by the Core Research for Evolutional Science and Technology (CREST) from Japan Science and Technology Agency (JST), and Grant-inAid for Scientific Research $(20300199,40359873)$ from the Japan Society for the Promotion of Science (JSPS).

\section{REFERENCES}

Bastiaansen, M., and Hagoort, P. (2006). Oscillatory neuronal dynamics during language comprehension. Prog. Brain Res. 159, 179-196. doi: 10.1016/S00796123(06)59012-0

Burton, M. W., Small, S. L., and Blumstein, S. E. (2000). The role of segmentation in phonological processing: an fMRI investigation. J. Cogn. Neurosci. 12, 679-690. doi: 10.1162/089892900562309

Canolty, R., Soltani, M., Dalal, S., Edwards, E., Dronkers, N., Nagarajan, S., et al. (2007). Spatiotemporal dynamics of word processing in the human brain. Front. Neurosci. 1:185. doi: 10.3389/neuro.01.1.1.014.2007

Chang, C.-C., and Lin, C.-J. (2011). LIBSVM: a library for support vector machines. ACM Trans. Intell. Sys. Technol. 2, 27:1-27:27. Available online at: http://www. csie.ntu.edu.tw/ cjlin/libsvm

Chang, E., Rieger, J., Johnson, K., Berger, M., Barbaro, N., and Knight, R. (2010). Categorical speech representation in human superior temporal gyrus. Nat. Neurosci. 13, 1428-1432. doi: 10.1038/nn.2641

Crone, N., Boatman, D., Gordon, B., and Hao, L. (2001a). Induced electrocorticographic gamma activity during auditory perception. Clin. Neurophysiol. 112, 565-582. doi: 10.1016/S1388-2457(00)00545-9

Crone, N., Hao, L., Hart, J., Boatman, D., Lesser, R., Irizarry, R., et al. (2001b). Electrocorticographic gamma activity during word production in spoken and sign language. Neurology 57, 2045. doi: 10.1212/WNL.57.11.2045

Crone, N. E., Miglioretti, D. L., Gordon, B., Sieracki, J. M., Wilson, M. T., Uematsu, S., et al. (1998). Functional mapping of human sensorimotor cortex with electrocorticographic spectral analysis. i. alpha and beta event-related desynchronization. Brain 121, 2271-2299. doi: 10.1093/brain/121.12.2271

DaSalla, C., Kambara, H., Sato, M., and Koike, Y. (2009). Single-trial classification of vowel speech imagery using common spatial patterns. Neural Netw. 22, 13341339. doi: 10.1093/brain/121.12.2271

Duffau, H., Capelle, L., Denvil, D., Gatignol, P., Sichez, N., Lopes, M., et al. (2003). The role of dominant premotor cortex in language: a study using intraoperative functional mapping in awake patients. Neuroimage 20, 1903-1914. doi: 10.1016/S1053-8119(03)00203-9

Giraud, A.-L., and Poeppel, D. (2012). Cortical oscillations and speech processing: emerging computational principles and operations. Nat. Neurosci. 15, 511-517. doi: 10.1038/nn.3063

Goto, T., Hirata, M., Umekawa, Y., Yanagisawa, T., Shayne, M., Saitoh, Y., et al. (2011). Frequency-dependent spatiotemporal distribution of cerebral oscillatory changes during silent reading: a magnetoencephalograhic group analysis. Neuroimage 54, 560-567. doi: 10.1016/j.neuroimage.2010.08.023

Haynes, J.-D., and Rees, G. (2006). Decoding mental states from brain activity in humans. Nat. Rev. Neurosci. 7, 523-534. doi: 10.1038/nrn1931

Herman, A. B., Houde, J. F., Vinogradov, S., and Nagarajan, S. S. (2013). Parsing the phonological loop: activation timing in the dorsal speech stream determines accuracy in speech reproduction. J. Neurosci. 33, 5439-5453. doi: 10.1523/JNEUROSCI.1472-12.2013

Hickok, G., and Poeppel, D. (2004). Dorsal and ventral streams: a framework for understanding aspects of the functional anatomy of language. Cognition 92, 67-99. doi: 10.1016/j.cognition.2003.10.011

Huang, J., Carr, T. H., and Cao, Y. (2002). Comparing cortical activations for silent and overt speech using event-related fMRI. Hum. Brain Mapp. 15, 39-53. doi: 10.1002/hbm. 1060

Leuthardt, E., Gaona, C., Sharma, M., Szrama, N., Roland, J., Freudenberg, Z., et al. (2011). Using the electrocorticographic speech network to control a brain-computer interface in humans. J. Neural Eng. 8, 036004. doi: $10.1088 / 1741-2560 / 8 / 3 / 036004$
Lotze, M., and Halsband, U. (2006). Motor imagery. J. Physiol. Paris 99, 386-395. doi: 10.1016/j.jphysparis.2006.03.012

McBride-Chang, C. (1996). Models of speech perception and phonological processing in reading. Child Dev. 67, 1836-1856. doi: 10.2307/1131735

Miller, K., Schalk, G., Fetz, E., den Nijs, M., Ojemann, J., and Rao, R. (2010). Cortical activity during motor execution, motor imagery, and imagerybased online feedback. Proc. Natl. Acad. Sci. U.S.A. 107, 4430. doi: 10.1073/pnas.0913697107

Obleser, J., Boecker, H., Drzezga, A., Haslinger, B., Hennenlotter, A., Roettinger, M., et al. (2006). Vowel sound extraction in anterior superior temporal cortex. Hum. Brain Mapp. 27, 562-571. doi: 10.1002/hbm.20201

Palmer, E. D., Rosen, H. J., Ojemann, J. G., Buckner, R. L., Kelley, W. M., and Petersen, S. E. (2001). An event-related fMRI study of overt and covert word stem completion. Neuroimage 14(1 pt 1), 182-193. doi: 10.1006/nimg.2001.0779

Pei, X., Barbour, D., Leuthardt, E., and Schalk, G. (2011). Decoding vowels and consonants in spoken and imagined words using electrocorticographic signals in humans. J. Neural Eng. 8, 046028. doi: 10.1088/1741-2560/8/4/ 046028

Pei, X., Leuthardt, E. C., Gaona, C. M., Brunner, P., Wolpaw, J. R., and Schalk, G. (2010). Spatiotemporal dynamics of electrocorticographic high gamma activity during overt and covert word repetition. Neuroimage 54, 2960-2972. doi: 10.1016/j.neuroimage.2010.10.029

Price, C. (2012). A review and synthesis of the first 20 years of PET and fMRI studies of heard speech, spoken language and reading. Neuroimage 62, 816-847. doi: 10.1016/j.neuroimage.2012.04.062

Saur, D., Kreher, B. W., Schnell, S., Kümmerer, D., Kellmeyer, P., Vry, M.-S., et al. (2008). Ventral and dorsal pathways for language. Proc. Natl. Acad. Sci. U.S.A. 105, 18035-18040. doi: 10.1073/pnas.0805234105

Sharma, N., Jones, P. S., Carpenter, T., and Baron, J.-C. (2008). Mapping the involvement of BA $4 \mathrm{a}$ and $4 \mathrm{p}$ during motor imagery. Neuroimage 41, 92-99. doi: 10.1016/j.neuroimage.2008.02.009

Vapnik, V. (1998). Statistical Learning Theory. New York, NY: Wiley.

Vigneau, M., Beaucousin, V., Herve, P.-Y., Duffau, H., Crivello, F., Houde, O., et al. (2006). Meta-analyzing left hemisphere language areas: phonology, semantics, and sentence processing. Neuroimage 30, 1414-1432. doi: 10.1016/j.neuroimage.2005.11.002

Wada, J., and Rasmussen, T. (1960). Intracarotid injection of sodium amytal for the lateralization of cerebral speech dominance. J. Neurosurg. 17, 266-282. doi: 10.3171/jns.1960.17.2.0266

Wildgruber, D., Ackermann, H., Klose, U., Kardatzki, B., and Grodd, W. (1996). Functional lateralization of speech production at primary motor cortex: a fMRI study. Neuroreport 7, 2791-2796. doi: 10.1097/00001756-199611040-00077

Zatorre, R. J., Evans, A. C., Meyer, E., and Gjedde, A. (1992). Lateralization of phonetic and pitch discrimination in speech processing. Science 256, 846-849. doi: 10.1126/science.1589767

Zatorre, R. J., Meyer, E., Gjedde, A., and Evans, A. C. (1996). PET studies of phonetic processing of speech: review, replication, and reanalysis. Cereb. Cortex 6, 21-30. doi: 10.1093/cercor/6.1.21

Conflict of Interest Statement: The authors declare that the research was conducted in the absence of any commercial or financial relationships that could be construed as a potential conflict of interest.

Received: 13 November 2013; accepted: 19 February 2014; published online: 07 March 2014.

Citation: Ikeda S, Shibata T, Nakano N, Okada R, Tsuyuguchi N, Ikeda K and Kato A (2014) Neural decoding of single vowels during covert articulation using electrocorticography. Front. Hum. Neurosci. 8:125. doi: 10.3389/fnhum.2014.00125 This article was submitted to the journal Frontiers in Human Neuroscience.

Copyright (C) 2014 Ikeda, Shibata, Nakano, Okada, Tsuyuguchi, Ikeda and Kato. This is an open-access article distributed under the terms of the Creative Commons Attribution License (CC BY). The use, distribution or reproduction in other forums is permitted, provided the original author(s) or licensor are credited and that the original publication in this journal is cited, in accordance with accepted academic practice. No use, distribution or reproduction is permitted which does not comply with these terms. 